\title{
Review Article \\ Clinical Relevance of HLA Antibody Monitoring after Kidney Transplantation
}

\author{
Christian Morath, ${ }^{1}$ Gerhard Opelz, ${ }^{2}$ Martin Zeier, ${ }^{1}$ and Caner Süsal ${ }^{2}$ \\ ${ }^{1}$ Department of Nephrology, University of Heidelberg, Im Neuenheimer Feld 162, 69120 Heidelberg, Germany \\ ${ }^{2}$ Department of Transplantation Immunology, Institute of Immunology, University of Heidelberg, Im Neuenheimer Feld 305, \\ 69120 Heidelberg, Germany
}

Correspondence should be addressed to Caner Süsal; caner.suesal@med.uni-heidelberg.de

Received 28 May 2014; Accepted 10 September 2014; Published 13 October 2014

Academic Editor: Mario Clerici

Copyright (c) 2014 Christian Morath et al. This is an open access article distributed under the Creative Commons Attribution License, which permits unrestricted use, distribution, and reproduction in any medium, provided the original work is properly cited.

In kidney transplantation, antibody-mediated allograft injury caused by donor HLA-specific antibodies (DSA) has recently been identified as one of the major causes of late graft loss. This paper gives a brief overview on the impact of DSA development on graft outcome in organ transplantation with a focus on risk factors for de novo alloantibody induction and recently published guidelines for monitoring of DSA during the posttransplant phase.

\section{Introduction}

The employment of new immunosuppressive strategies in the late 1980s and 1990s and their efficacy to control T-cell alloimmunity led to a striking decrease in the occurrence of T-cell-mediated acute rejection episodes. Simultaneously, our shortcomings in controlling antibody-mediated rejection (AMR) were revealed and the importance of chronic AMR has become apparent. Mainly two developments contributed to a deeper understanding of antibody-mediated allograft injury: (1) the recognition that deposition of the complement split product C4d (especially in peritubular capillaries of the kidney allograft) may indicate antibodymediated allograft injury and (2) the association of donor HLA-specific antibodies (DSA) detected by highly sensitive techniques with inferior outcome of kidney transplants $[1,2]$. Recent investigations indicate that more than $60 \%$ of late kidney graft losses are due to antibody-mediated humoral tissue injury, and there has been increasing evidence that HLA antibodies are responsible for graft losses not only in kidney but also in other solid organ transplantations [35]. Therefore, HLA antibodies and their association with AMR have become the main focus of research in organ transplantation.

\section{Tissue Damage Caused by Donor HLA-Specific Antibodies}

Early after transplantation, acute AMR occurs in about 1 to $6 \%$ of patients; however, this frequency may increase up to 21 to $55 \%$ in patients who had detectable DSA already before transplantation and who received desensitization therapy [6-8]. Persistence or reemergence of DSA that were detectable already before transplantation is associated with poor allograft outcome [9]. Weak pretransplant DSA have been associated with rather subtle types of graft damage, often leading to delayed graft function [10]. It is well known that early damage can later on translate into chronic (antibodymediated) changes, most likely because the structure of the endothelium is injured and new antigenic epitopes are expressed on the surface of transplanted tissue. During later phases after transplantation, insufficient immunosuppression and stimulation of the memory cell response by inflammatory events can support the development of de novo DSA against antigenic structures and result in failure of the transplanted organ due to antibody-mediated organ injury.

Additional antibodies that are discussed in the evolution of chronic AMR are MICA antibodies, angiotensin II type 1 receptor activating antibodies, and other antiendothelial cell 
antibodies [11-13]. The exact impact of these antibodies on the outcome of kidney and other organ transplants needs, however, yet to be determined.

In this overview, we focus on the impact of de novo HLA alloantibodies that are detected after kidney transplantation.

\section{Donor HLA-Specific Antibodies Become the Most Important Parameter in the Diagnosis of Antibody-Mediated Kidney Allograft Rejection}

Currently, features of AMR in the biopsy together with the detection of a circulating DSA are required for the histological diagnosis of antibody-mediated kidney graft rejection. In addition, evidence of antibody interaction with the vascular endothelium must be present, either by C4d positivity or microvascular inflammation (peritubular capillaries and/or glomerulitis) [14]. Of note, in the latest update of the BANFF classification (BANFF 2013), detection of C4d-positivity in peritubular capillaries is no longer considered a prerequisite for the diagnosis of AMR. Instead, moderate microvascular inflammation or even the demonstration of AMR-specific gene transcripts together with circulating DSA is accepted as diagnostic criterions for the diagnosis of AMR. In particular, in chronic AMR, C4d may often be negative (C4d-negative AMR). Before the introduction of highly sensitive antibody detection techniques, such as the Luminex single antigen bead (L-SAB) assay, there was often no DSA detectable in patients with chronic AMR due to the low levels of antibody. L-SAB now allows the detection of DSA with high sensitivity. Only recently, Wiebe et al. reported that even weakly reactive, L-SAB-detected de novo DSA measured at the low positivity cut-off of $300 \mathrm{MFI}$ is predictive of graft survival [15]. Everly et al. confirmed this observation, with the exception that they used the higher cut-off of 1,000 MFI [16].

\section{Risk Factors for the Development of (De Novo) Donor HLA-Specific Antibodies}

Risk factors for the development of de novo DSA, AMR, and graft loss are not uniformly described. In many patients with late antibody-mediated graft loss, even when HLA class I alloantibodies are detectable, circulating HLA class II de novo DSA are considered to be mainly responsible for rejection. Therefore, most authors believe that specifically HLA class II mismatches (not only HLA-DR but also HLA-DQB, DQA, and DP mismatches) confer an increased risk for late graft loss [15-17]. Due to the strong linkage disequilibrium between the DR and DQB or DQA (but not DP) gene loci, two DR mismatches often indeed represent 6 mismatches that are relevant for induction of DSA. Additional risk factors for the de novo development of DSA and subsequent occurrence of (chronic) AMR are younger age, deceased donor kidney transplantation, presence of HLA antibodies before transplantation, nonadherence to immunosuppressive medication (see below), and insufficient immunosuppression or drug minimization $[4,16,17]$.
TABLE 1: Risk factors for the de novo development of DSA.

\begin{tabular}{lc}
\hline Risk factor & Reference \\
\hline Retransplantation & {$[21]$} \\
HLA antibodies before transplantation & {$[16,21]$} \\
Young age (18-35 years old) & {$[16]$} \\
Deceased donor transplantation & {$[16]$} \\
DR, DQ mismatch & {$[16,22]$} \\
Nonadherence & {$[4,15]$} \\
Insufficient immunosuppression & {$[17]$} \\
Inflammation (i.e., infection) & {$[18,19]$} \\
(Subclinical) T-cell-mediated rejection & {$[7,15]$} \\
\hline
\end{tabular}

Most importantly, early inflammatory events, such as infections, minor surgery, trauma, and particularly early (acute) T-cell-mediated rejection episodes, often precede de novo DSA development and AMR [18-20]. Even subclinical cellular rejection may lead to HLA antibody development with an increased risk for antibody-mediated allograft injury in subsequent years [7].

Table 1 gives an overview on the risk factors for de novo DSA development.

\section{Graft Survival after Development of $D e$ Novo Donor HLA-Specific Antibodies}

Hidalgo et al. found DSA in $37 \%$ of patients who had an indication biopsy 7 days to 31 years after transplant [23]. In particular, de novo DSA, which made up $60 \%$ of all DSA and were directed against HLA class II antigen mismatches of the donor, were associated with strongly impaired graft survival: within 5 years from DSA detection, $50 \%$ of the patients in the study of Hidalgo lost their grafts. Wiebe et al. found a 10 -fold increase in graft loss in patients who developed de novo DSA, with a $40 \%$ lower graft survival rate 10 years after DSA development compared to patients without de novo DSA [15]. Everly et al. reported on a $24 \%$ graft loss rate 3 years after de novo occurrence of DSA [16]. However, in all these studies, low numbers of patients with graft loss were investigated. We compared in sera of 51 patients with graft loss that were obtained prior to graft failure and in sera of matched controls with functioning grafts the incidence of $d e$ novo DSA and non-DSA [24]. Patients with graft loss showed a higher incidence of both DSA and non-DSA than patients without graft loss.

\section{C1q-Binding HLA Antibodies}

A recent development is the introduction of solid-phase assays that allow the distinction of complement-binding (C1q assay) or complement-activating (C4d assay) HLA antibodies from HLA antibodies that do not bind or activate complement.

While two early pediatric studies that investigated the use of the $\mathrm{Clq}$ assay for the detection of de novo DSA after transplantation found conflicting results $[25,26]$, a recent landmark study by Loupy et al. demonstrated that 
the occurrence after transplantation of complement-binding DSA in a cohort of 1,016 patients transplanted between January 1, 2005, and January 1, 2011, was associated with adverse outcomes [21]. The 5-year graft survival rate in patients who developed complement-binding DSA (de novo and persistent/reemerging) was 54\%, strikingly lower than the $93 \%$ rate in patients with DSA that were not complementbinding or the $94 \%$ rate in patients without any DSA. The higher graft loss rate in patients with complement-binding DSA was attributable to a higher rate of AMR, especially in the patients who developed complement-binding DSA de novo after kidney transplantation. Interestingly, pretransplant complement-binding DSA did not have the same predictive values since about half of the patients lost these antibodies after transplantation.

\section{Nonadherence and Reduction of Immunosuppression as Major Contributors to Late Graft Loss}

Einecke et al. reported in 2009 that antibody-mediated microcirculation injury is one of the leading causes of late graft loss, together with death with a functioning graft, recurrent renal disease, and interstitial fibrosis/tubular atrophy (of unknown origin) [3]. Chronic AMR is found more frequently in patients who are nonadherent to immunosuppressive medication or in whom immunosuppression was reduced or withdrawn for other reasons, for example, conversion to calcineurin-inhibitor-free or steroid-free immunosuppressive protocols, recurrent infection, or malignancy $[4,15,17$, 21]. Patients at high risk for nonadherence are young adults who are in the transition phase from pediatric to adult renal services. Other risk factors are previous nonadherence, psychiatric disorders, substance abuse, and insufficient socioeconomic support, but also adverse effects of immunosuppressive medication.

In a recent publication, 64\% of graft losses in a selected patient cohort with indication biopsies were found attributable to (antibody-mediated) rejection [4]. Importantly, about half of the patients with rejection-associated graft loss were identified as nonadherent. In the study of Wiebe et al., who investigated the evolution of HLA antibodies after transplantation, de novo DSA were found to appear at a mean of 4.6 years after transplantation, and the prevalence of de novo DSA after 10 years was $20 \%$ in adherent as compared to a remarkable $60 \%$ in nonadherent graft recipients [15]. Of note, in this study, patients were thoroughly screened to exclude any preexisting antibodies. Patients were considered to have de novo DSA only when posttransplant DSA occurred after the current and all historic sera at the time of transplantation were negative at a $300 \mathrm{MFI}$ cut-off and when patients showed no evidence of AMR in a 6month protocol biopsy. With this definition, no de novo DSA were detectable at 6 months and only a $2 \%$ incidence of de novo DSA was recorded at 1 year.

Not only nonadherence of the patient to immunosuppressive medication but also reduction of immunosuppression by the physician may lead to adverse outcomes after kidney transplantation. Opelz and Döhler hinted already in 2008 on the problem of "insufficient immunosuppression" as a major cause of graft loss. In an analysis of more than 25,000 kidney transplant recipients, they showed that, in patients with good graft function at year one, discontinuation or reduction after the first posttransplant year of cyclosporine, tacrolimus, or mycophenolate mofetil below certain threshold levels was associated with significantly reduced graft survival during the subsequent years [27].

Insufficient immunosuppression may also occur during immunosuppressive minimization (tapering) or calcineurininhibitor-avoidance trials. In a recent study, 14 of 61 patients (23\%) that were converted from cyclosporine to everolimus at 3-4.5 months after transplantation developed DSA, compared to only 7 of 65 patients (11\%) who continued on cyclosporine [17]. Eight patients on everolimus, but only 2 patients on cyclosporine, developed AMR. It needs to be mentioned that in this study many patients off cyclosporine, unfortunately, were also off steroids which might have biased the results. Nevertheless, these data indicate that particularly patients with reduction or discontinuation of immunosuppressive medication should be screened rigorously for the occurrence of HLA antibodies and antibody-mediated allograft injury. In patients with DSA, minimization of immunosuppression should altogether be avoided. Nonadherence to immunosuppressive medication and insufficient immunosuppression does not only lead to the development of de novo DSA but has also significant impact when DSA already are present [15]. Wiebe et al. found nonadherence in $100 \%$ of patients with de novo DSA and acute graft dysfunction, whereas the rate was only $6 \%$ in patients with de novo DSA but stable graft function.

\section{What Do the Guidelines of the Transplantation Society (TTS) Tell Us about Posttransplant Antibody Monitoring?}

Several important issues are covered by the consensus guidelines that have been published in early 2013 [28].

(1) Posttransplant screening for DSA is recommended for all patient groups in the early postoperative period, however, at different time points dependent on the pretransplant risk of the patient for AMR. In "low risk" patients, who were not sensitized to HLA before transplantation and who received their first allograft, the possible presence of DSA should be examined at least once in the period from 3 to 12 months after transplantation. In "intermediate risk" patients, who were antibody-negative at the time of transplantation but had DSA in previous testing, DSA should be examined already during the first month. No further testing is recommended for both groups during the first year, unless (i) there is a change in immunosuppression, (ii) nonadherence is suspected, (iii) graft dysfunction occurs, or (iv) the patient is transferred to a remote outside center. (2) If DSA are present at any time, a biopsy should be performed, and if the biopsy result is positive, treatment of AMR is recommended. In DSA-positive "high risk" patients and in desensitized crossmatch-positive "very high risk" patients, in addition to DSA monitoring, 
a biopsy is recommended for all patients during the first 3 months after transplantation. Even if the biopsy result is negative in these two groups but there are rapidly increasing DSA or if the biopsy shows subclinical rejection, treatment of AMR should be initiated. In the absence of AMR, DSA should be monitored and immunosuppression maintained at higher levels. (3) Beyond year one, no routine DSA monitoring is recommended for the four risk groups, except when one of the abovementioned four conditions occurs. Of note, a minority of members within the guidelines group supported HLA antibody monitoring at least once a year in all patients to rule out antibody-mediated allograft injury at its earliest stage. (4) If DSA are detected beyond year 1, patients should be treated and monitored essentially as described above for the first year after transplantation.

It needs to be mentioned that the consensus guidelines of TTS were published before Loupy and coworkers published their seminal paper on the impact of complement-binding HLA alloantibodies on kidney graft survival.

\section{Conclusions}

In summary, despite its known technical limitations [29], the highly sensitive L-SAB assay appears to be a very useful tool for posttransplant monitoring of HLA antibodies and for surveillance of AMR. HLA antibodies that occur de novo after transplantation and that are complement-binding/activating denote the highest risk for AMR and graft loss. However, also the recurrence of preexisting antibodies after transplantation or the development of de novo non-DSA may confer an increased risk for graft loss.

\section{Conflict of Interests}

The authors declare that there is no conflict of interests regarding the publication of this paper.

\section{References}

[1] H. E. Feucht, H. Schneeberger, G. Hillebrand et al., "Capillary deposition of C4d complement fragment and early renal graft loss," Kidney International, vol. 43, no. 6, pp. 1333-1338, 1993.

[2] P. I. Terasaki, "Humoral theory of transplantation," The American Journal of Transplantation, vol. 3, no. 6, pp. 665-673, 2003.

[3] G. Einecke, B. Sis, J. Reeve et al., "Antibody-mediated microcirculation injury is the major cause of late kidney transplant failure," American Journal of Transplantation, vol. 9, no. 11, pp. 2520-2531, 2009.

[4] J. Sellarés, D. G. de Freitas, M. Mengel et al., "Understanding the causes of kidney transplant failure: the dominant role of antibody-mediated rejection and nonadherence," The American Journal of Transplantation, vol. 12, no. 2, pp. 388-399, 2012.

[5] G. J. Berry, M. M. Burke, C. Andersen et al., "The 2013 international society for heart and lung transplantation working formulation for the standardization of nomenclature in the pathologic diagnosis of antibody-mediated rejection in heart transplantation," Journal of Heart and Lung Transplantation, vol. 32, no. 12, pp. 1147-1162, 2013.
[6] C. Lefaucheur, A. Loupy, G. S. Hill et al., "Preexisting donorspecific HLA antibodies predict outcome in kidney transplantation," Journal of the American Society of Nephrology, vol. 21, no. 8, pp. 1398-1407, 2010.

[7] P. Amico, G. Hönger, M. Mayr, J. Steiger, H. Hopfer, and S. Schaub, "Clinical relevance of pretransplant donor-specific HLA antibodies detected by single-antigen flow-beads," Transplantation, vol. 87, no. 11, pp. 1681-1688, 2009.

[8] J. M. Gloor, J. L. Winters, L. D. Cornell et al., "Baseline donorspecific antibody levels and outcomes in positive crossmatch kidney transplantation," American Journal of Transplantation, vol. 10, no. 3, pp. 582-589, 2010.

[9] K. Klein, C. Süsal, S. M. Schäfer et al., "Living donor kidney transplantation in patients with donor-specific HLA antibodies enabled by anti-CD20 therapy and peritransplant apheresis," Atherosclerosis Supplements, vol. 14, no. 1, pp. 199-202, 2013.

[10] C. Süsal, B. Döhler, M. Sadeghi, J. Ovens, and G. Opelz, "HLA antibodies and the occurrence of early adverse events in the modern era of transplantation: a collaborative transplant study report," Transplantation, vol. 87, no. 9, pp. 1367-1371, 2009.

[11] Y. Zou, P. Stastny, C. Süsal, B. Döhler, and G. Opelz, "Antibodies against MICA antigens and kidney-transplant rejection," The New England Journal of Medicine, vol. 357, no. 13, pp. 1293-1300, 2007.

[12] N. L. Reinsmoen, C.-H. Lai, H. Heidecke et al., "Antiangiotensin type 1 receptor antibodies associated with antibody mediated rejection in donor HLA antibody negative patients," Transplantation, vol. 90, no. 12, pp. 1473-1477, 2010.

[13] T. Mohanakumar, J. C. Waldrep, M. Phibbs, G. Mendez-Picon, A. M. Kaplan, and H. M. Lee, "Serological characterization of antibodies eluted from chronically rejected human renal allografts," Transplantation, vol. 32, no. 1, pp. 61-66, 1981.

[14] M. Haas, B. Sis, L. C. Racusen et al., "Banff 2013 meeting report: inclusion of $\mathrm{C} 4 \mathrm{~d}$-negative antibody-mediated rejection and antibody-associated arterial lesions," The American Journal of Transplantation, vol. 14, no. 2, pp. 272-283, 2014.

[15] C. Wiebe, I. W. Gibson, T. D. Blydt-Hansen et al., "Evolution and clinical pathologic correlations of de novo donor-specific HLA antibody post kidney transplant," American Journal of Transplantation, vol. 12, no. 5, pp. 1157-1167, 2012.

[16] M. J. Everly, L. M. Rebellato, C. E. Haisch et al., "Incidence and impact of de Novo donor-specific alloantibody in primary renal allografts," Transplantation, vol. 95, no. 3, pp. 410-417, 2013.

[17] L. Liefeldt, S. Brakemeier, P. Glander et al., "Donor-specific HLA antibodies in a cohort comparing everolimus with cyclosporine after kidney transplantation," American Journal of Transplantation, vol. 12, no. 5, pp. 1192-1198, 2012.

[18] I. Katerinis, K. Hadaya, R. Duquesnoy et al., "De novo antiHLA antibody after pandemic H1N1 and seasonal influenza immunization in kidney transplant recipients," The American Journal of Transplantation, vol. 11, no. 8, pp. 1727-1733, 2011.

[19] J. E. Locke, A. A. Zachary, D. S. Warren et al., "Proinflammatory events are associated with significant increases in breadth and strength of HLA-specific antibody," American Journal of Transplantation, vol. 9, no. 9, pp. 2136-2139, 2009.

[20] C. Wiebe and P. Nickerson, "Posttransplant monitoring of de novo human leukocyte antigen donor-specific antibodies in kidney transplantation," Current Opinion in Organ Transplantation, vol. 18, no. 4, pp. 470-477, 2013.

[21] A. Loupy, C. Lefaucheur, D. Vernerey et al., "Complementbinding anti-HLA antibodies and kidney-allograft survival," 
The New England Journal of Medicine, vol. 369, no. 13, pp. 12151226, 2013.

[22] M. C. S. Freitas, L. M. Rebellato, M. Ozawa et al., "The role of immunoglobulin-G subclasses and Clq in de novo HLADQ donor-specific antibody kidney transplantation outcomes," Transplantation, vol. 95, no. 9, pp. 1113-1119, 2013.

[23] L. G. Hidalgo, P. M. Campbell, B. Sis et al., "De novo donorspecific antibody at the time of kidney transplant biopsy associates with microvascular pathology and late graft failure," The American Journal of Transplantation, vol. 9, no. 11, pp. 25322541, 2009.

[24] C. Süsal, B. Döhler, A. Ruhenstroth et al., "Association of kidney graft loss with posttransplant presence of strong HLA antibodies detected by Luminex single antigen testing. A Collaborative Transplant Study Report," in Proceedings of the TTS Congress, Berlin, Germany, 2012, abstract no. MON.CO16.01.

[25] S. M. Sutherland, G. Chen, F. A. Sequeira, C. D. Lou, S. R. Alexander, and D. B. Tyan, "Complement-fixing donor-specific antibodies identified by a novel Clq assay are associated with allograft loss," Pediatric Transplantation, vol. 16, no. 1, pp. 12-17, 2012.

[26] F. Ginevri, A. Nocera, P. Comoli et al., "Posttransplant de novo donor-specific HLA antibodies identify pediatric kidney recipients at risk for late antibody-mediated rejection," The American Journal of Transplantation, vol. 12, no. 12, pp. 33553362, 2012.

[27] G. Opelz and B. Döhler, "Effect on kidney graft survival of reducing or discontinuing maintenance immunosuppression after the first year posttransplant," Transplantation, vol. 86, no. 3, pp. 371-376, 2008.

[28] B. D. Tait, C. Süsal, H. M. Gebel et al., "Consensus guidelines on the testing and clinical management issues associated with HLA and Non-HLA antibodies in transplantation," Transplantation, vol. 95, no. 1, pp. 19-47, 2013.

[29] P. Gombos, G. Opelz, S. Scherer et al., "Influence of test technique on sensitization status of patients on the kidney transplant waiting list," American Journal of Transplantation, vol. 13, no. 8, pp. 2075-2082, 2013. 


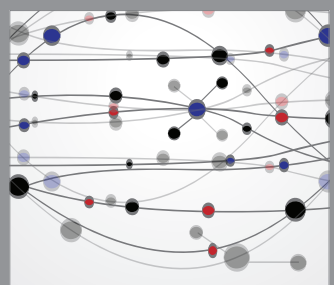

The Scientific World Journal
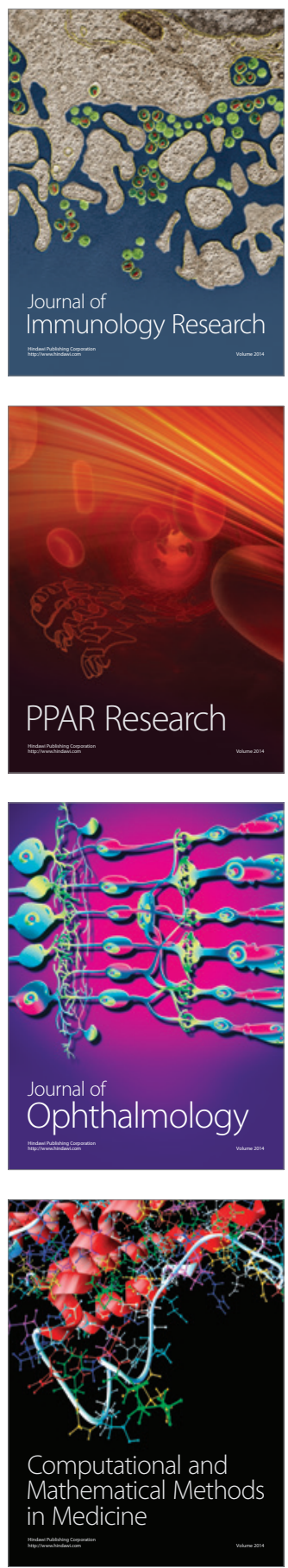

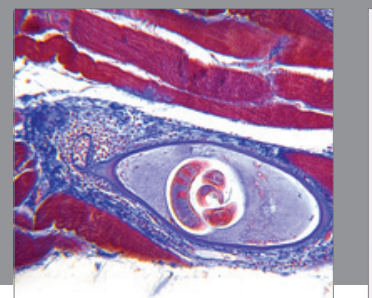

Gastroenterology

Research and Practice
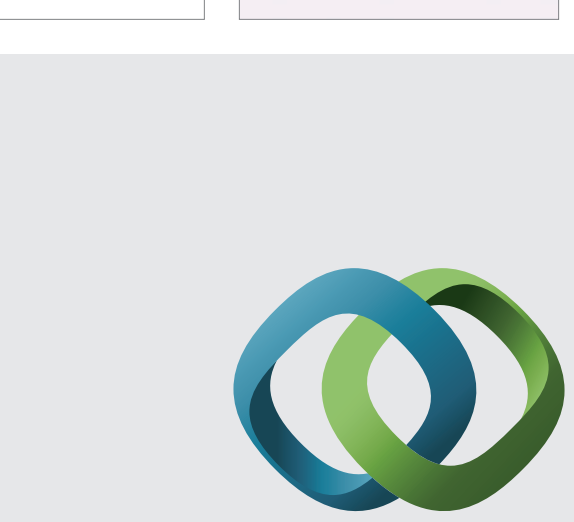

\section{Hindawi}

Submit your manuscripts at

http://www.hindawi.com
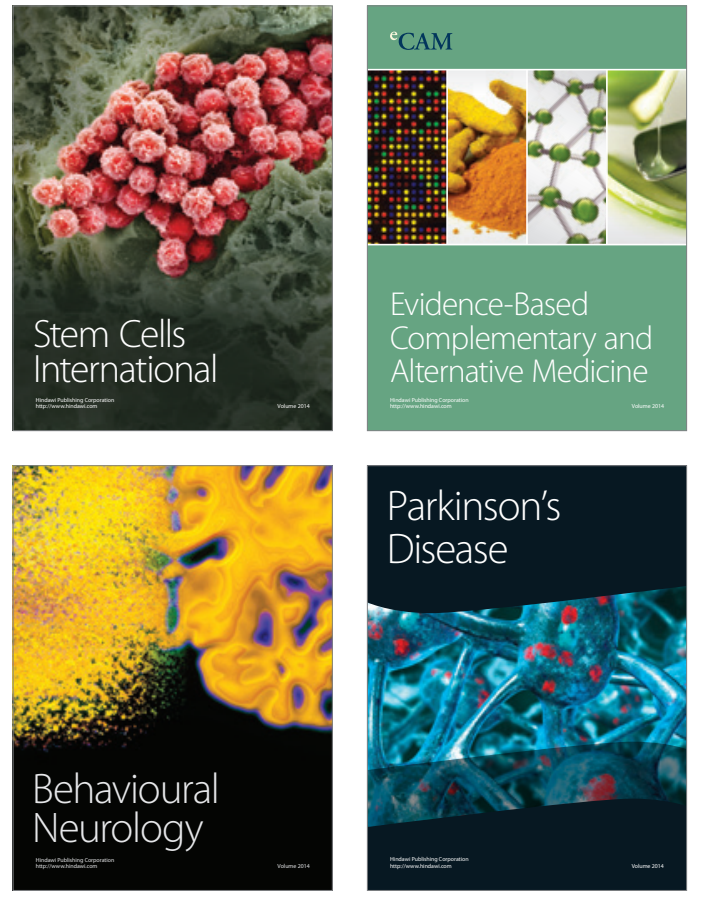
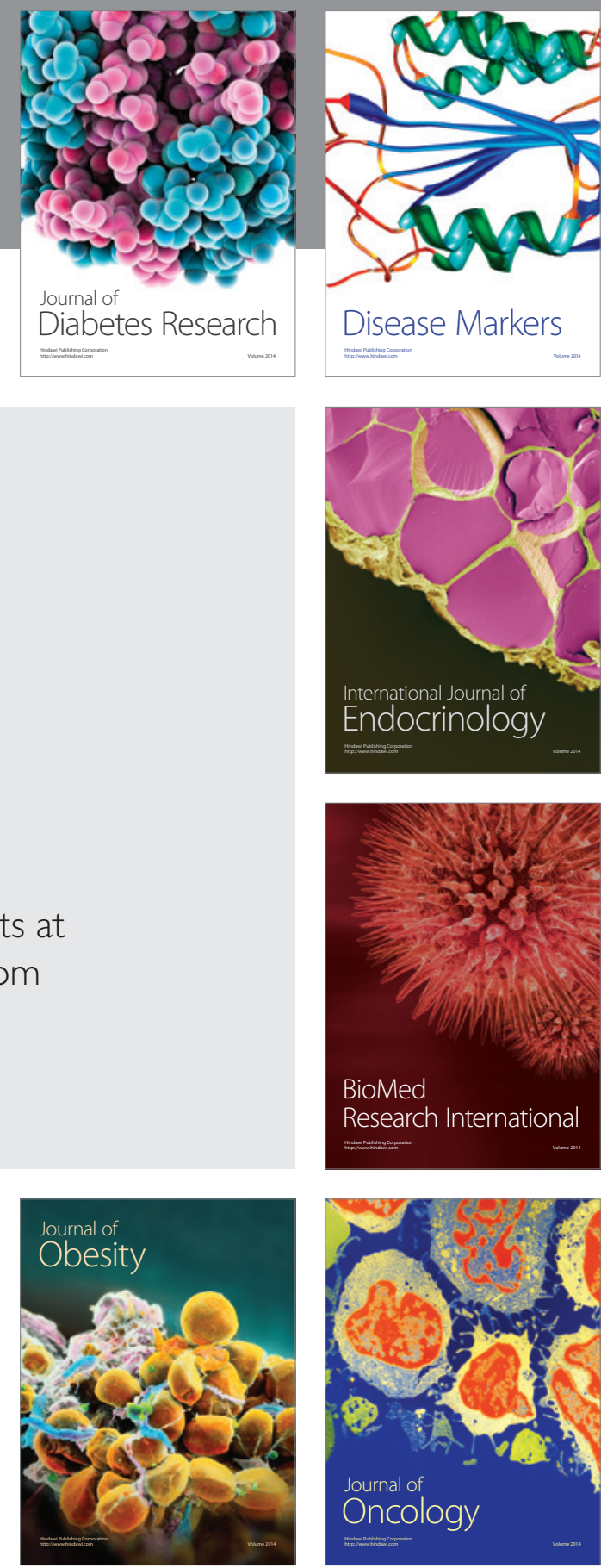

Disease Markers
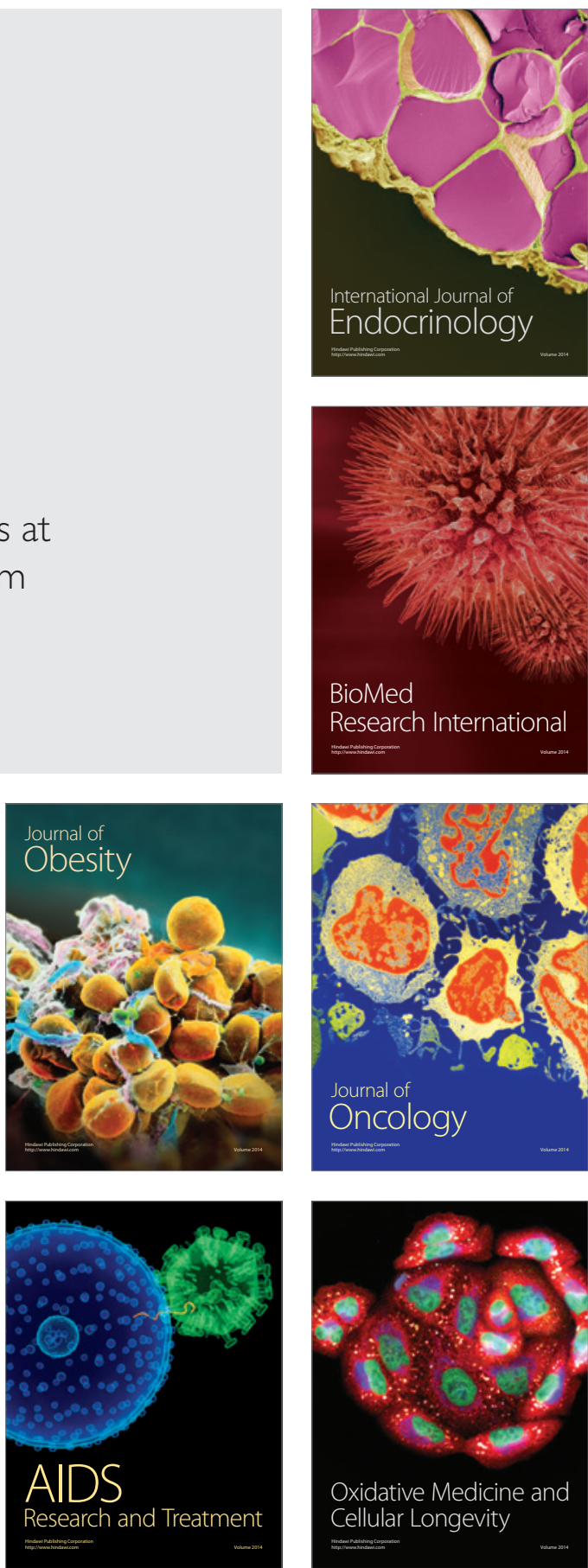